analysis reveals that the majority of differentially methylated genes in the enhancer region are involved in the cancer and focal adhesion pathway. In addition, the pathways in cancer and PI3K-Akt signaling are significantly enriched in the differentially methylated open chromatin loci. Significant differentially methylated enhancer and open chromatin loci, OPLAH cg26256223 (hypermethylated open chromatin) and LYN cg08621168 (hypomethylated enhancer) were selected for further validation. qPCR analysis further confirmed the decrease of OPLAH gene expression level and vice versa for the LYN gene

Conclusions This is the first insight on the enhancers and open chromatins methylation profile in Malaysian CRC patients. The new knowledge from this study can be utilized to further increase our understanding of CRC methylomics, particularly on the enhancers and open chromatins. The functional roles of OPLAH cg26256223 and LYN cg08621168 warrant future investigations

\section{IDDF2019-ABS-0307 LONG NON-CODING RNA TMPO-AS1 REGULATES OESOPHAGEAL SQUAMOUS CELL CARCINOMA METASTASES THROUGH ACTIVATING GLI1 BY MAINTAINING LAP2A EXPRESSION}

Xiao-Jing Luo*, Jia Liu, Huai-Qiang Ju, Rui-Hua Xu. State Key Laboratory of Oncology in South China, Collaborative Innovation Center for Cancer Medicine, Sun Yat-sen University Cancer Center, China

\subsection{6/gutjnl-2019-IDDFabstracts.69}

Background Long non-coding RNAs (lncRNA) have been found to play important regulatory roles in cancer development and progression. However, functional lncRNAs and their downstream mechanisms remain largely unknown of oesophageal squamous cell carcinoma (OSCC) metastases. We aimed to identify lncRNAs that regulate OSCC metastases and investigate their downstream mechanisms.

Methods Small interfering RNA library was built of the top 50 overexpressed lncRNAs in OSSC according to TCGA database. Transwell migration assay was performed to identify the lncRNA that markedly affected cell migration. TMPO-AS expression was validated by $\mathrm{qPCR}$ in patient tissues and OSSC cell lines. Gain and loss of function of TMPO-AS 1 were performed in transwell migration and invasion assays in vitro. Lung and lymph node metastases models were built with short hairpin RNA knockdown in vivo. RNA pull-down and RNAase protection assay (RPA) followed by $\mathrm{qPCR}$ analysis identified the RNA-RNA interaction. Downstream mechanisms were examined by regular molecular biological methods.

Results We identified TMPO-AS 1 as a key regulator of OSSC metastases. TMPO-AS 1 expression was upregulated in OSSC tumor tissues compared to adjacent normal tissues and positively correlated with the mRNA expression of TMPO (LAP2), its sense coding gene. Knockdown of TMPO-AS 1 significantly inhibited OSSC cells migration and invasion in vitro and attenuated metastases in two different animal models in vivo. Overexpression of TMPO- $A S_{1}$ showed contrary effects. RNA pull-down identified the interaction between TMPO-AS mRNA and LAP2a mRNA. RPA assay further confirmed $T M P O-A S_{1}$ 's protective effect on $L A P 2 a$ mRNA. Western blotting found that knockdown of TMPO-AS ${ }_{1}$ decreased the expression of LAP2a without affecting LAP2b. Ectopic expression of LAP2a after TMPO-AS $S_{1}$ knockdown rescued the adverse effect on cell migration and invasion. By regulating the expression of its sense coding gene LAP2, TMPO-AS maintained the levels of LAP2a, which in turn activated Hedgehog signaling transcription factor $\mathrm{GLI}_{1}$ and its downstream target SNAIL, therefore promoting OSSC progression. Conclusions TMPO-AS $S_{1}$ acts as an essential regulator in OSSC metastases by interacting with $L A P 2 a$ mRNA and maintaining its levels, which activates the LAP $2 a-G_{1} I_{1}-S N A I L$ axis and facilitates OSSC metastases.

\section{IDDF2019-ABS-0312 THE LANDSCAPE OF RECURRENT NONCODING MUTATIONS IN COLORECTAL CANCERS}

${ }^{1}$ Nurul-Syakima Ab Mutalib*, ${ }^{1}$ Ryia Illani Mohd Yunos, ${ }^{1}$ Najwa Farhah Mohd Yusof, ${ }^{1}$ Muhiddin Ishak, ${ }^{1}$ Sazuita Saidin, ${ }^{2}$ Isa Mohamed Rose, ${ }^{3}$ Luqman Mazlan, ${ }^{3}$ Ismail Sagap, ${ }^{1}$ Rahman Jamal. 'UKM Medical Molecular Biology Institute (UMBI), National University of Malaysia, Malaysia; ${ }^{2}$ Department of Pathology, Faculty of Medicine, National University of Malaysia, Malaysia; ${ }^{3}$ Department of Surgery, Faculty of Medicine, National University of Malaysia, Malaysia

\subsection{6/gutjnl-2019-IDDFabstracts.70}

Background Colorectal cancer (CRC) is among major cancer worldwide, and it has become evident that the identification of driver mutations is fundamental to understanding carcinogenesis. Although genes frequently mutated in CRC have been identified, those pursuits for driver mutations have mainly focused on the coding genome. The noncoding somatic mutation landscape remains unexplored. Hence, this study aims to characterize the landscape of noncoding somatic mutations in CRC.

Methods Genomic DNA was extracted from 36 cancerous colonic tissues and subjected to whole genome sequencing (WGS). Blood DNA served as the germline control. The sequencing data were aligned to hg19 using Burrow-Wheeler Aligner (BWA), somatic variants were called using muTect2 and the noncoding mutations were prioritized using funseq2. Pathway enrichment analysis was performed using DAVID, and gene expression data were retrieved from Firebrowse.

Results We identified 72,890 recurrent noncoding alterations, which were altered in at least 2 patients. Focusing on the distal regulatory modules (DRMs), the majority (83.2\%) of the alterations were identified in transcription factor binding peaks (TFP), followed by Segway/ChromHMM-predicted enhancers (8.39\%) and DNase I hypersensitive sites (DHS) (3.68\%). In addition, $0.56 \%$ alterations were discovered in the long intergenic noncoding RNAs (lincRNAs) and $0.02 \%$ in transcription factor bound motifs in peak regions (TFM). MAFK| chr4:50489-63382 and WRNIP1|chr6:292554-300803 are the most frequently altered TFPs (9/36), while drm chr17:21514800-21518300 is the most frequently altered enhancers (8/36). There was a modest upregulation of MAFK $(\log 2$ RSEM $=0.997)$ and WRNIP1 $(\log 2$ RSEM $=1.45)$ gene expression in cancer compared to the normal based on 626 CRCs from COADREAD TCGA dataset. LincRNA (ENSG00000238261.3[BX004987.5]) alteration was identified in $8 / 36$ of patients. We also show the commonality of pathways targeted by coding and noncoding mutations, demonstrated by TP53, APC, and KRAS, which regulates Wnt and MAPK signaling, the crucial pathways in colorectal carcinogenesis.

Conclusions This study provides an enhanced understanding of colorectal carcinogenesis and describes the advantages of 